Review

\title{
Recent Progress in Optical Fiber Sensors Based on Brillouin Scattering at University of Ottawa
}

\author{
Xiaoyi BAO and Liang CHEN \\ Fiber Optics Group, Physics Department, University of Ottawa, Ottawa, ON, K1N 6N5, Canada \\ *Corresponding author: Xiaoyi BAO_E-mail: xbao@uottawa.ca
}

\begin{abstract}
The distributed sensor is proven to be a powerful tool for civil structural and material process monitoring. Brillouin scattering in fiber can be used as point sensors or distributed sensors for measurement of temperature, strain, birefringence and vibration over centimeters (Brillouin grating length) for point sensor or the pulse length for the distributed sensor. Simultaneous strain and temperature measurement with a spatial resolution of $20 \mathrm{~cm}$ is demonstrated in a Panda fiber using Brillouin grating technique with the temperature accuracy and strain accuracy of $0.4{ }^{\circ} \mathrm{C}$ and $9 \mu \varepsilon$. This technique can also be used for distributed birefringence measurement. For Brillouin optical time domain analysis (BOTDA), we have developed a new technique to measure differential Brillouin gain instead of Brillouin gain itself. This technique allows high precision temperature and strain measurement over long sensing length with sub-meter spatial resolution: $50-\mathrm{cm}$ spatial resolution for $50-\mathrm{km}$ length, using return-to-zero coded optical pulses of BOTDA with the temperature resolution of $0.7{ }^{\circ} \mathrm{C}$, which is equivalent to strain accuracy of $12 \mu \varepsilon$. For over $50-\mathrm{km}$ sensing length, we proposed and demonstrated frequency-division-multiplexing (FDM) and time-division-multiplexing (TDM) based BOTDA technique for $75-\mathrm{km}$ and $100-\mathrm{km}$ sensing length without inline amplification within the sensing length. The spatial resolution of $2 \mathrm{~m}(100 \mathrm{~km})$ and Brillouin frequency shift accuracy of $1.5 \mathrm{MHz}$ have been obtained for TDM based BOTDA and 1-m resolution $(75 \mathrm{~km})$ with Brillouin frequency shift accuracy of $1 \mathrm{MHz}$ using FDM based BOTDA. The civil structural health monitoring with BOTDA technique has been demonstrated.
\end{abstract}

Keywords: Brillouin scattering, distributed sensor, Brillouin grating, temperature, strain, birefringence

\section{Distributed fiber sensors based on Brillouin scattering}

For over two decades, distributed optical fiber sensors based on Brillouin scattering have gained much interest for their potential capabilities of monitoring temperature [1] and strain [2] in civil and structural engineering, environmental monitoring, and geotechnical engineering. Brillouin scattering occurs as a result of refractive-index fluctuations caused by acoustic waves resulted from thermally generated sound waves, and such thermal agitation is capable of scattering incident lightwaves with shifted frequencies. Stimulated Brillouin scattering (SBS) enhances the Brillouin scattering in a Brillouin optical time domain analysis (BOTDA) [3-8] with intense signal and better spatial resolution comparing with a spontaneous scattering based Brillouin optical time domain reflectometry (BOTDR) [9-11]. At present, there are two schemes to realize a BOTDA, including Brillouin gain [3-5] and Brillouin loss [6-8]. In a Brillouin-gain-based

Received: 23 September 2010 / Revised version: 9 November 2010

(C) The Author(s) 2011.This article is published with open access at Springerlink.com 
sensor, the pulsed light is used as a pump and has a higher frequency over continuous-wave (CW) light, so that the CW light (Stokes wave) experiences gain through SBS process; on the contrary, in the case of Brillouin loss, the CW light used as a pump experiences loss after propagating in the sensing fiber and the pulsed light gains energy whose frequency is lower than that of the $\mathrm{CW}$ light.

In the experiment, two lightwaves, the pump and the probe signals, are launched into a fiber in a counter-propagating configuration. The simultaneous presence of Stokes and the pump waves generates a beat signal that reinforces acoustic wave in the fiber when light beam frequency difference equals to Brillouin frequency. The scattering of the pump is then enhanced, leading to its depletion and the input probe beam is amplified. The probe wave is also called Stokes wave as it corresponds to the frequency downshifted signal. Brillouin spectrum can be recorded by tuning the frequency difference between the pump and Stokes waves.

For a short-range fiber, with low pump depletion in Brillouin gain and small excess amplification on the probe pulse in the Brillouin loss, two techniques give the similar results. However, in a long-ranged BOTDA system via Brillouin gain, the maximum input power of CW light is severely limited by the pump depletion of pulsed pump light due to SBS threshold, which decreases Brillouin signal and also induces measurement errors at the far end of the fiber, and thus shortens the measureable sensing length. In a Brillouin-loss-based system, the excess amplification on the probe pulse induced by $\mathrm{CW}$ pump can also distort Brillouin spectrum and produce measurement errors at the far end of the sensing fiber. However, increasing the probe pulse power aggravates much less on excess amplification effect than that induced by higher pump power, so that a high-power probe pulse could be used to improve the signal-to-noise ratio (SNR) with minimum pump power. As the $\mathrm{CW}$ is detected in DC (direct current) mode with high contrast due to the low pump power and low DC offset, hence the broadband detection maintains the high spatial resolution.

\section{Performance parameters}

Various parameters need to be considered when comparing Brillouin sensor systems. First, one has to keep in mind that the sensor must be implemented in the field. It must then be simple to install and fast in the sensing operation. Second, all of the sensor performance parameters are critical. Those are the spatial resolution, which indicates the smallest detectable event size over the fiber length, the frequency resolution, which is the smallest Brillouin frequency shift that can be measured (here we refer the temperature or strain resolution), and the measurement range, which is the longest length over which the sensor can make an accurate data acquisition. Typical distributed sensor based on Brillouin scattering has the spatial resolution of $1 \mathrm{~m}$ which is limited by phonon lifetime $[12,17]$.

\section{Spatial resolution improvement to sub-meters (<phonon lifetime)}

Recent research effort has been directed towards reducing spatial resolution to centimeters, which is practical for structural monitoring applications. Two solutions were successfully proposed and demonstrated in recent years to achieve sub-meter spatial resolution.

The first solution is to avoid spectral spreading of Brillouin spectrum due to the broadband pulse signal by generating a pre-pumping of the acoustic wave using weak CW leakage (DC level of the un-modulated pulse signal) of the pulse, then making the interaction of pump and probe on this pre-pumped acoustic wave [12-16]. This solution combines the advantage of an ideally narrow Brillouin gain spectrum with high spatial resolution of very short pulses. Spatial resolution down to $10 \mathrm{~cm}$ has been demonstrated using this scheme with 
$1 \mathrm{~ns}$ optical pulse. This is remarkable, as it was believed that $1-\mathrm{m}$ spatial resolution would be the best that could ever be achieved due to the finite lifetime of the acoustic phonons [17]. By using this idea a small crack $(1 \mathrm{~cm})$ has been detected on the optical ground wire [14].

The second solution is to generate frequencydithered signals that propagate in opposite directions [18]. If the dithering frequencies are identical, the frequency difference between two waves is constant only at periodic locations along the fiber. At these points stimulated Brillouin interaction is very efficient and localized. The position of the interaction point can be scanned along the fiber by varying the dithering frequency, although the scanning time can be very long [19], and centimeter spatial resolution was demonstrated using this scheme.

Most recently, a differential pulsewidth pair Brillouin optical time-domain analysis (DPP-BOTDA) for high spatial resolution sensing has been proposed [20] to measure differential Brillouin gain instead of Brillouin gain itself. This scheme employs two different long pulses (a few tens of nanoseconds) with a small pulse-width difference (1 ns or less) to map Brillouin gain spectrum (BGS) of the sensing fiber. The differential BGS can be obtained by subtraction between the two BGSs, and its spatial resolution is determined by the pulsewidth difference of two long pulses. The DPP-BOTDA provides several advantages over conventional BOTDA: 1) narrowband BGS (a few tens $\mathrm{MHz}$ ) and high spatial resolution (centimeters) can be obtained simultaneously over tens of kilometers and 2) differential BGS provides stronger signal intensity and thus better SNR than that of directly using a narrow pulse in BOTDA when the pulse-width difference of two long pulses equals to the narrow pulse width.

In DPP-BOTDA the differential Brillouin gain is realized in the electronic domain, i.e. the signal is subtracted in intensity domain, so the measurement takes twice as long, and the large pulse introduces the gain saturation over the long sensing length. The further exploration of this technology in optical field domain is based on coherent interaction of the Brillouin gain and loss via optical differential parametric amplification (ODPA) [21]. Because it is not a Brillouin gain or loss as demonstrated in DPP-BOTDA, ODPA provides a narrowed parametric Brillouin gain spectrum and short measurement time simultaneously. Because ODPA involves Stokes wave and anti-Stokes wave, one must be careful in handling Brillouin frequency difference between Stokes wave and anti-Stokes wave.

\section{Stokes and anti-Stokes frequency shift}

To a very good approximation spontaneous Brillouin scattering in the fiber describes the phenomenon that when a laser of angular frequency $\omega_{0}$ is propagating inside an optical fiber, two resonant back scattered light waves at angular frequencies $\omega_{0} \pm \Omega_{B}$ will be observed due to the interaction of light wave with an acoustic wave of speed $V_{a}$, where $\Omega_{B}$ is defined as Brillouin angular frequency. The up-shifted light $\omega_{0}+\Omega_{B}$ is called anti-Stokes wave and the down-shifted light $\omega_{0}-\Omega_{B}$ is called Stokes wave. Aforementioned description of Brillouin scattering effect in fiber is wide spread [22], however, it is unfortunately true only up to the first order ratio between sound and light speeds [23]. We can also view Brillouin scattering on the basis of relativistic Doppler effect [24].

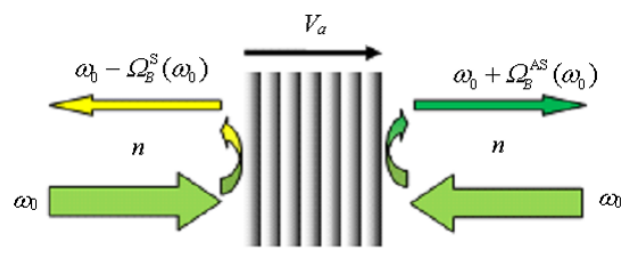

Fig. 1 Schematic diagram for the Doppler effect. 
As plotted in Fig. 1 we describe Brillouin scattering in a view that light of angular frequency $\omega_{0}$ is incident on a moving acoustic wave at speed $V_{a}$ and then a head-on collision creates a reflected

$$
\omega_{0}+\Omega_{B}^{\mathrm{AS}}\left(\omega_{0}\right)=\omega_{0} \sqrt{\frac{1+\frac{n\left(\omega_{0}\right) V_{a}}{c}}{1-\frac{n\left(\omega_{0}\right) V_{a}}{c}}} \sqrt{\frac{1+\frac{n\left(\omega_{0}+\Omega_{B}^{\mathrm{AS}}\left(\omega_{0}\right)\right) V_{a}}{c}}{1-\frac{n\left(\omega_{0}+\Omega_{B}^{\mathrm{AS}}\left(\omega_{0}\right)\right) V_{a}}{c}}}
$$

where $n\left(\omega_{0}\right)$ is the refractive index of the fiber at frequency $\omega_{0}$, and $c$ is the speed of light in vacuum. Similarly the tail-end collision induces a reflected light (Stokes) with an angular frequency shifted downward by (2). Obviously Stokes angular

$$
\omega_{0}-\Omega_{B}^{\mathrm{S}}\left(\omega_{0}\right)=\omega_{0} \sqrt{\frac{1-\frac{n\left(\omega_{0}\right) V_{a}}{c}}{1+\frac{n\left(\omega_{0}\right) V_{a}}{c}}} \sqrt{\frac{1-\frac{n\left(\omega_{0}-\Omega_{B}^{\mathrm{S}}\left(\omega_{0}\right)\right) V_{a}}{c}}{1+\frac{n\left(\omega_{0}-\Omega_{B}^{\mathrm{S}}\left(\omega_{0}\right)\right) V_{a}}{c}}} .
$$

\section{Differential Brillouin gain to achieve centimeter spatial resolution}

Different pulse pair (DPP) to form BOTDA [20] can be realized with two different pulses of pulse width $\tau$ and $\tau+\delta \tau$ as probe wave interacts with the pump wave to generate the Brillouin loss signal. When the pump wave is scanned across the Brillouin spectrum of the fiber, we can get Brillouin loss spectrum at every fiber location for two pulse widths. Because of small pulse width difference $\delta \tau$, the difference in Brillouin loss is negligible for the same strain or temperature. When the stress or temperature varies at a specific fiber location the difference of the Brillouin loss at this location will be quite different on zero signal background. Figure 2 shows the experimental plot of Brillouin loss spectrum with 50-ns pulse (Fig. 2(a)) and pulse pair of 50/49 ns (Fig. 2(b)) to detect 50-cm strain section for the rise time of $0.2 \mathrm{~ns}$ (equivalent to $20 \mathrm{~cm}$ ). Hence, the spatial resolution has been improved by a factor of 25 with DPP-BOTDA. The uncertainty for measuring the Brillouin frequency shift is $3 \mathrm{MHz}$, frequency $\Omega_{B}^{\mathrm{S}}\left(\omega_{0}\right)$ and anti-Stokes angular frequency $\Omega_{B}^{\mathrm{AS}}\left(\omega_{0}\right)$ must be different according to the solutions of (1) and (2). We note as well that (1) and (2) are being written down for the first time in a dispersive medium here.

which is equivalent to 50 micro-strain.

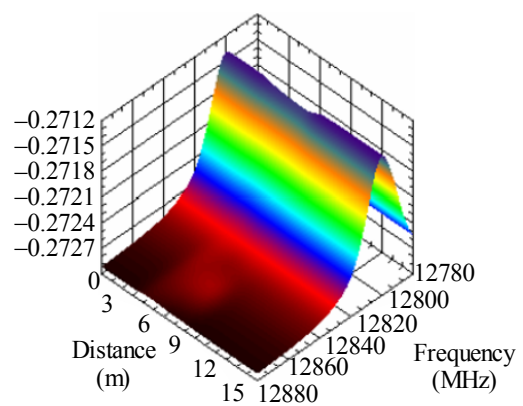

(a)

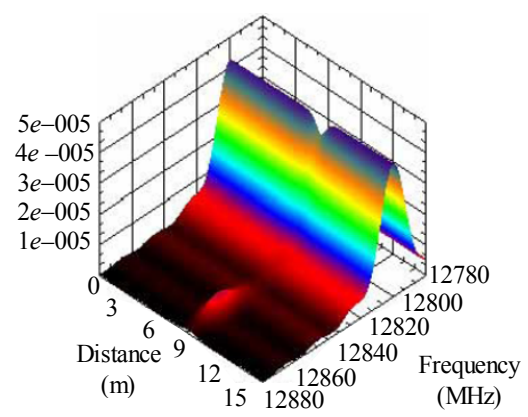

(b)

Fig. 2 3D BOTDA spectra along the sensing fiber for a 0.2 -m-length fiber section with $60-\mathrm{MHz}$ Brillouin frequency shift from 12,800 MHz: (a) conventional BOTDA using 50-ns pulse light and (b) DPP-BOTDA using 50-ns and 49-ns pulse pair [20]. 


\section{Optical differential parametric amplification in BOTDA}

ODPA uses coherent interaction of Brillouin gain and loss to reduce the pump depletion, instead of using two continuous waves to perform Brillouin gain and loss process as that in [25]. Two long pulses with a small pulse width difference at the Stokes and anti-Stokes frequencies respectively, interact with a $\mathrm{CW}$ sensing wave through two counter-propagating acoustic waves, creating Brillouin gain and loss at the same position simultaneously. At the overlapping region of two long pulses, the gain and loss can eliminate each other when they are well balanced, which is

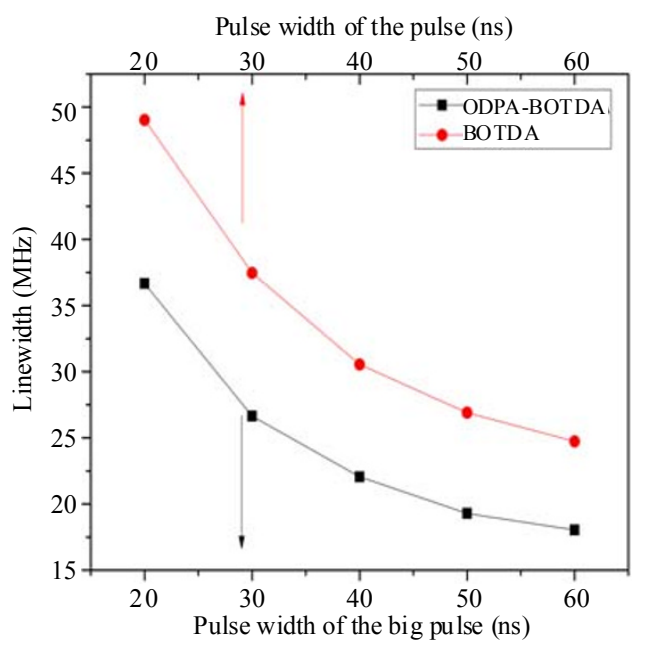

(a) "similar" to the subtraction process in DPP-BOTDA in electric domain. However, here it is realized in coherent optical field domain via ODPA with half of measurement time. Figure 3 shows the scheme of ODPA-BOTDA, and the process can be described by (3) [21] via slowly varying amplitude approximation and adopting the same notations and symbols as that in [22], i.e. CGS units are adapted.

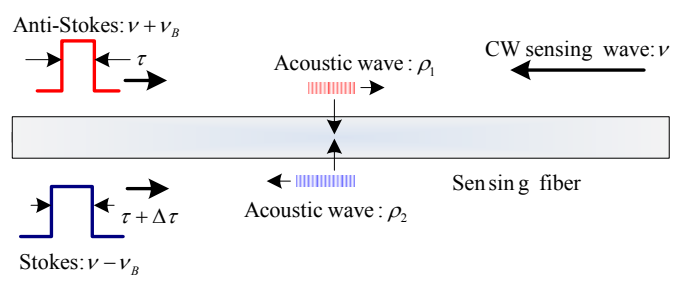

Fig. 3 Schematic diagram of ODPA-BOTDA.

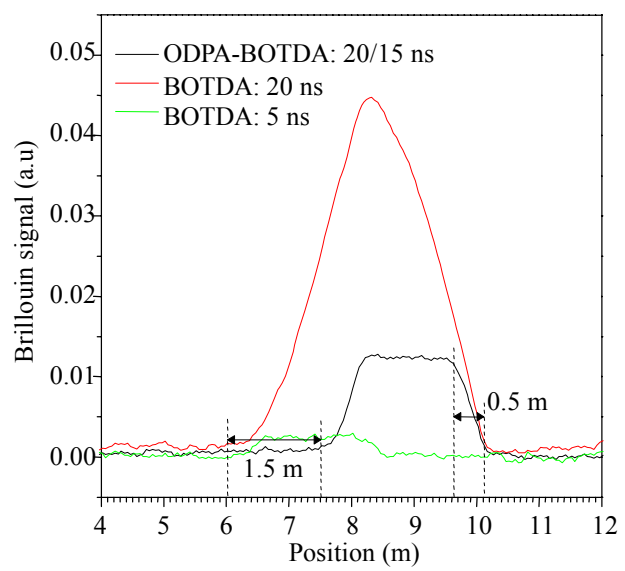

(b)

Fig. 4 (a) Brillouin spectrum width of BOTDA (20-60 ns pulse) and ODPA-BOTDA sensor with 20/18-60/58 ns pulse pair based on (7) and (b) time domain signal of ODPA-BOTDA vs. BOTDA.

$$
\left\{\begin{array}{l}
-\frac{\partial A_{1}}{\partial z}+\frac{n}{c} \frac{\partial A_{1}}{\partial t}=\frac{i \omega_{1} \gamma_{e}}{2 n c \rho_{0}} \tilde{\rho}_{1} A_{2}+\frac{i \omega_{1} \gamma_{e}}{2 n c \rho_{0}} \tilde{\rho}_{2}^{*} A_{3}-\frac{1}{2} \alpha A_{1} \\
\frac{\partial A_{2}}{\partial z}+\frac{n}{c} \frac{\partial A_{2}}{\partial t}=\frac{i \omega_{2} \gamma_{e}}{2 n c \rho_{0}} \tilde{\rho}_{1}^{*} A_{1}-\frac{1}{2} \alpha A_{2} \\
\frac{\partial A_{3}}{\partial z}+\frac{n}{c} \frac{\partial A_{3}}{\partial t}=\frac{i \omega_{3} \gamma_{e}}{2 n c \rho_{0}} \tilde{\rho}_{2} A_{1}-\frac{1}{2} \alpha A_{3} \\
\left(-2 i \Omega_{1}+\Gamma_{B}\right) \frac{\partial \tilde{\rho}_{1}}{\partial t}+\left(\Omega_{B}^{2}-\Omega_{1}^{2}-i \Omega_{1} \Gamma_{B}\right) \tilde{\rho}_{1}=\frac{\gamma_{e} q_{1}^{2}}{4 \pi} A_{1} A_{2}^{*} \\
\left(-2 i \Omega_{2}+\Gamma_{B}\right) \frac{\partial \tilde{\rho}_{2}}{\partial t}+\left(\Omega_{B}^{2}-\Omega_{2}^{2}-i \Omega_{2} \Gamma_{B}\right) \tilde{\rho}_{2}=\frac{\gamma_{e} q_{2}^{2}}{4 \pi} A_{3} A_{1}^{*}
\end{array}\right.
$$

where $\alpha$ is the attenuation coefficient of fiber, and $\Gamma_{B}$ is the inverse phonon lifetime. In the parametric amplification process, the interaction between optical fields $E_{1}, E_{2}$, and acoustic field $\tilde{\rho}_{1}$ is a 
Brillouin loss process. $E_{1}$ also interacts with $E_{3}$ via acoustic field $\tilde{\rho}_{2}$, which is a Brillouin gain process. The effect of Brillouin loss process and Brillouin gain process on optical field $E_{1}$ is a coherent process of field addition rather than intensity addition. In (3) it is clear that in equation for $A_{1}$, $E_{1}$ is associated with $E_{2}$ via $A_{2}$ and $E_{3}$ via $A_{3}$ simultaneously. Due to the well balanced energy transfer between Brillouin gain process and Brillouin loss process, Brillouin spectral width is much narrower than that in Brillouin gain or loss as shown in Fig. 4(a).

2-m strain section is measured with ODPA-BOTDA technique shifted by $1.5 \mathrm{~m}$ that corresponds to 15 -ns pulse of 20/15-ns pulse pair shown in Fig. 4(b). The spatial resolution of ODPA-BOTDA sensor is $0.5 \mathrm{~m}$ and is much smaller than that of BOTDA sensor with 20-ns probe and the signal contrast is 5 times stronger than that in 5 -ns BOTDA.

\section{Simultaneous temperature and strain sensing using distributed Brillouin sensor}

One problem with the implementation of Brillouin scattering based sensing systems in the field is the sensitivity of Brillouin frequency shift to both strain and temperature. This leads to ambiguity in the measurement, as one does not know whether the frequency shift is caused by the change of strain or temperature. In a laboratory environment the temperature is essentially constant and its effects can generally be neglected when measuring strain. In many field situations this is not the case.

An early solution to this problem proposed the use of two fibers placed adjacent to one another, in which one fiber was isolated from any strain effect [7]. The isolated fiber would be used to monitor temperature, while the other fiber would measure the effect of both strain and temperature. Recent innovative approaches to measuring strain and temperature simultaneously have been developed.
By combining Landau-Placzek ratio with frequency shift, temperature and strain were determined simultaneously (in spontaneous Brillouin scattering regime) at a spatial resolution of $40 \mathrm{~m}$ [26]. By using the same principle, an improved system has been reported which achieved a temperature resolution of $4{ }^{\circ} \mathrm{C}$, a strain resolution of 290 micro-strain, and a spatial resolution of $10 \mathrm{~m}$ for a sensing length of $15 \mathrm{~km}$ with LEAF fiber [27]. The demonstration of Brillouin gain for the power and Brillouin shift provides better performance for simultaneous temperature and strain measurement interpretation over the standard communication fiber, it gives 3-m spatial resolution for the temperature resolution of $3{ }^{\circ} \mathrm{C}$ and strain resolution of $180 \mu \varepsilon$ [28].

Centimeter resolution has been achieved with simultaneous temperature and strain sensing using Brillouin loss based distributed sensor with polarization maintaining fibers (PMF) and photonic crystal fibers (PCF).

Simultaneous temperature and strain sensing using PMF can be realized with Stokes power (intensity), linewidth, and Brillouin frequency. Unlike single mode fiber where the intensity fluctuation is overwhelmed by polarization mode dispersion induced polarization change in the fiber. The intensity fluctuation in PMF is only caused by light source power fluctuations. If the intensity change of light source is negligible, the intensity change caused by temperature and strain can be measured accurately [29]. Therefore the dependence of Brillouin spectrum width on the temperature and strain can be measured accurately. Thus three parameters (Brillouin frequency, Stokes power, and Brillouin spectral width) can be used for temperature and strain measurement simultaneously [30]. Table 1 gives the performance of simultaneous temperature and strain measurement with three kinds of PMFs using Brillouin power, linewidth, and peak frequency. 
Table 1 Uncertainty of temperature and strain calculated with measured Brillouin frequency represented by $F$, power represented by $P$, and bandwidth represented by $B$.

\begin{tabular}{c|c|c|c|c|c|c|c}
\hline \multirow{2}{*}{ Property } & \multicolumn{3}{|c|}{ Panda } & \multicolumn{3}{c|}{ Bow Tie } & Tiger \\
\cline { 2 - 9 } & $F-P$ & $F-B$ & $P-B$ & $F-P$ & $F-B$ & $P-B$ & $F-P$ \\
\hline Uncertainty of temperature $\left({ }^{\circ} \mathrm{C}\right)$ & 8 & 2 & 38 & 4 & 3 & 38 & 16 \\
\hline Uncertainty of strain $(\mu \varepsilon)$ & 153 & 39 & 135 & 237 & 126 & 195 & 598 \\
\hline Maximum error of $\Delta T\left({ }^{\circ} \mathrm{C}\right)$ & 10 & 4 & 58 & 12 & 7 & 137 & 78 \\
\hline Maximum error of $\Delta \varepsilon(\mu \varepsilon)$ & 331 & 82 & 249 & 741 & 490 & 1096 & 1308 \\
\hline $\operatorname{rms}(\Delta T)\left({ }^{\circ} \mathrm{C}\right)$ & 2 & 2 & 40 & 8 & 4 & 73 & 38 \\
\hline $\operatorname{Rms}(\Delta \varepsilon)(\mu \varepsilon)$ & 221 & 43 & 149 & 414 & 152 & 567 & 926 \\
\hline
\end{tabular}

In PCFs with solid silica core the guiding mechanism is the same as conventional single mode fiber, except the effective cladding index is the average of air and silica refractive indices. The solid silica core with a Ge-doped center region can increase the nonlinear refractive index of the core and create a smaller mode field diameter. As a result, the Brillouin spectrum of PCF shows multiple peaks with comparable intensities [31] with the main peak and several sub-resonance peaks due to guided acoustic modes. The temperature coefficients for the main resonance and a peak originating from a higher-order guided longitudinal acoustic mode in PCF with a partially graded Ge-doped core were identified for simultaneous temperature and strain measurement using their Brillouin frequency shifts only. It allows high temperature and strain measurement accuracy with spatial resolution of 20 $\mathrm{cm}[32]$.

\section{Offset locking of DFB lasers and bias control of optical modulator}

Recently, a distributed Brillouin sensor based on offset locking of two DFB lasers has been developed to measure temperature and strain changes of large structures [33]. In this offset locking [34] based setup, an optical phase locked loop is applied to lock and tune the beat frequency of pump and probe DFB lasers. Thus, two offset locked DFB lasers can have similar phase performance as those highly stabilized lasers such as Nd: YAG lasers.

The offset locking technique [34] was used to stabilize the frequency of a millimeter-wave sub carrier, except a PID (proportional integral derivative) controller in hardwire was employed to lock the beat frequency, which provided a fast response determined by optical delay line. The frequency tuning was achieved by tuning the delay time of optical delay line as shown in Fig. 5. The only limitation to the tuning frequency range was the delay range. To keep the optical modulator at high extinction ratio, we used a lock-in amplifier to stabilize the bias drift at minimum DC level [35]. This technique gave signal to noise ratio of over $35 \mathrm{~dB}$ for Brillouin frequency measurement accuracy of $0.5 \mathrm{MHz}$ for $1-\mathrm{m}$ spatial resolution over $2-\mathrm{km}$ fiber length.

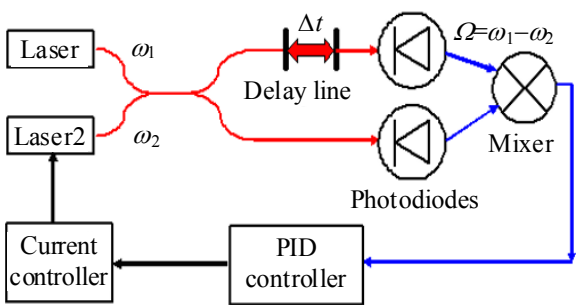

Fig. 5 Laser frequency offset locking system.

\section{Long range distributed sensor with BOTDA}

The sensing length is limited by Brillouin gain saturation which limits the pump power and probe power to the sensing fiber, hence a large pulse width has been used for the long sensing length in order to maintain signal to noise ratio (SNR) above $20 \mathrm{~dB}$ for high strain resolution of about $20 \mu \varepsilon$. By using DPP-BOTDA technique over $25-\mathrm{km}$ sensing length, sub-meter $(50 \mathrm{~cm})$ spatial resolution is demonstrated for the first time [36] with the strain resolution of $20 \mu \varepsilon$ as shown in Fig. 6. The results were achieved with single pulse; limitation of the sensing length is the modulation instability (MI) [36]. As probe power of $400 \mathrm{~mW}$ is used, pump power has dropped to $0.1 \mathrm{~mW}$ for $25-\mathrm{km}$ dispersion shifted fiber. The spatial resolution is limited by broadband electronic noise and strain or temperature resolution is limited by gain saturation caused by SBS in DPP signal (Fig. 6). This means an optimal SNR can be achieved with a specific pulse pair to achieve maximum 
differential Brillouin gain over the entire sensing length.

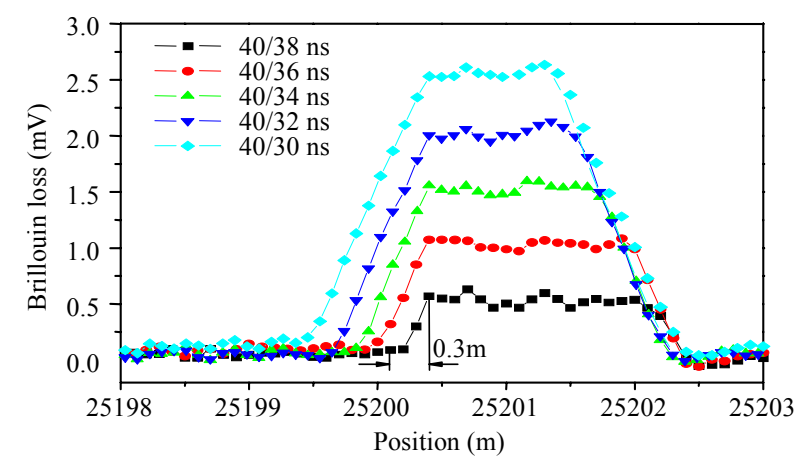

Fig. 6 Differential Brillouin loss signals for different pulses pairs (40/38 ns-40/30 ns) [35].

\section{0. $R Z$ coded BOTDA to detect two closely located strain sections}

To achieve longer sensing length $(>30 \mathrm{~km})$, the coded pulse must be considered and it must be combined with DPP-BOTDA technique. Coding techniques have already been used for BOTDR [37-39] and BOTDA [40, 41]. All of them have used non-return-to-zero (NRZ) coded pulse which has strong pattern dependence due to nonlinear amplification of stimulated Brillouin scattering from continuous " 1 "s, as the signals do not return to zero in every bit, unlike return-to-zero (RZ) coded pulse which returns to zero in every bit. Because of the optical filter effect from nonlinear amplification of different frequency components of optical pulses, NRZ signal tends to produce error in the form of broadening the stress length at the falling edge of time domain waveform, which causes the difficulty in identifying two closely located stress sections, as well as enlarging small stress length. While the RZ coded pulse returns to zero in every bit, it preserves the input pulse shape due to twice bandwidth of that in NRZ and measures small stress length and consecutive stress sections. Because of small distortion in RZ-BOTDA waveform, we can use DPP-BOTDA technique to improve spatial resolution to centimetres over 50-km LEAF fiber at low Brillouin gain [42, 43]. We stretched two 2.3-m sections separated by $1.5-\mathrm{m}$ loose fiber as shown in
Fig. 7. The pulse width is 40 ns. Figure 8 shows the measured Brillouin frequency shift (BFS) versus position for RZ coded pulse of 512 bits (Fig. 8(b)) and NRZ coded pulse of 512 bits (Fig. 8(a)) respectively. Two strained sections are clearly distinguished in RZ coded pulses, which is not in the case of NRZ coded pulse. The pulse broadening in NRZ results in a smeared boundary, which makes the falling edge have less dependence on Brillouin frequency change to Brillouin loss, while RZ maintains the pulse shape, the spatial resolution and less cross talk between boundaries of two different strain or temperature sections.

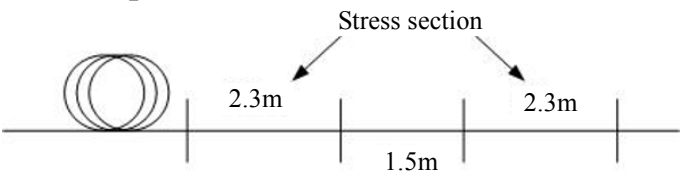

Fig. 7 Spatial distribution of two stress sections at the end of fiber.

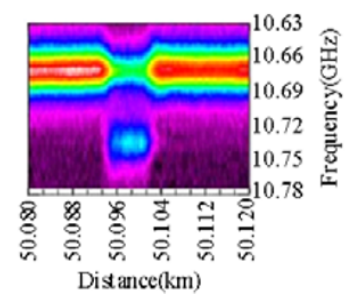

(a)

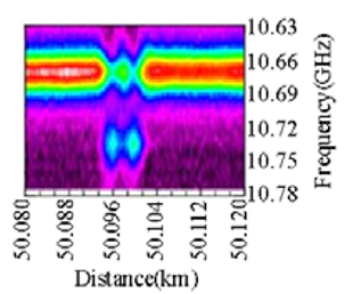

(b)
Fig. 8 Two stress sections of $2.3 \mathrm{~m}$ with $1.5-\mathrm{m}$ separation for NRZ (a) and RZ pulse (b) of BOTDA [43].

The best spatial resolution achieved with RZ coded pulse is illustrated in Fig. 9 [42], and 50-cm spatial resolution with strain resolution of $8 \mu \varepsilon$, which is equivalent to a $0.7-\mathrm{MHz}$ Brillouin frequency shift. This is the 1 st sub-meter spatial resolution with sub-MHz strain resolution over 50-km length.

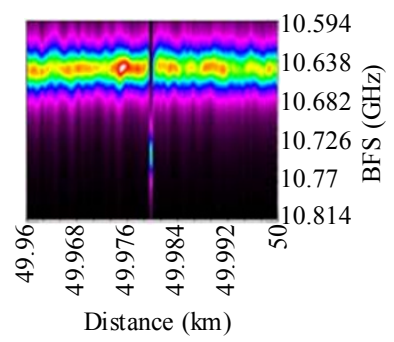

Fig. 9 Differential Brillouin spectrum of 5-ns RZ coded pulses for 50-cm stress length [43]. 


\section{Frequency- and time- division-multiplexing for BOTDA}

For the fiber length of $100 \mathrm{~km}$ the dynamic range of Brillouin gain or loss signal is $40 \mathrm{~dB}$, which includes fiber loss of $20 \mathrm{~dB}$. True signal change due to pump wave and probe wave in BOTDA is only $20 \mathrm{~dB}$. This means detectable change is $0.2 \mathrm{~dB} / \mathrm{km}$ and $0.002 \mathrm{~dB} / 10 \mathrm{~m}$. For any change induced on 100-m spatial resolution, the detect ability must be better than $4.6 \times 10^{-4}$, which means SNR must be better than $40 \mathrm{~dB}$. It is about $1-{ }^{\circ} \mathrm{C}$ temperature resolution. Even if we add erbium doped fiber amplifier (EDFA) or Raman amplifier, the signal to noise ratio will not be improved, as those amplifiers only increase signal level at the cost of adding additional noise to sensor system.

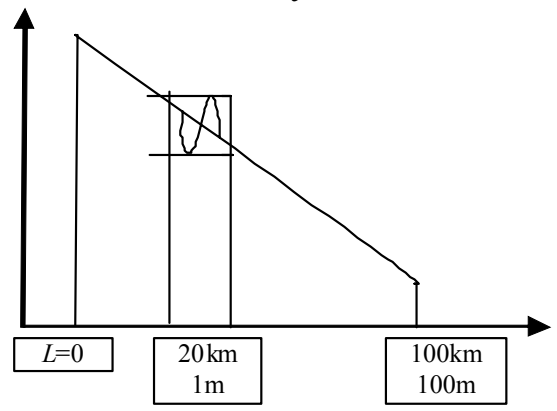

Fig. 10 Enlarged dynamic range over effective sensing length.

One can separate the dynamic range of $40 \mathrm{~dB}$ in frequency domain and time domain over the limited sensing length for the measurement. In frequency domain it is frequency-division-multiplexing (FDM) BOTDA for different sections of the fibers and at one measurement, the sensing range is much shorter than entire sensing length. This means Brillouin interaction happens only within the sensing section with maximum Brillouin gain, while the remaining sections are only subjected to fiber loss. In this way the spatial resolution and the strain or temperature resolution are determined by the measured fiber section and among $100 \mathrm{~m}$ it is much easier to detect a 1-m change instead of detecting a 1-m change over $100 \mathrm{~km}$ as shown in two scales of Fig. 10. The total sensing length will be the combination of multiple series of effective lengths which are added in frequency domain or time domain via time-division multiplexing (TDM).

\subsection{FDM based BOTDA}

FDM-BOTDA is based on different types of fibers with different Brillouin frequencies, so that Brillouin gain in each fiber section can be maximized instead of being minimized in BOTDA approach, and the effective fiber length has been reduced to that section of the fiber rather than entire sensing length. When the frequency difference of pump wave and probe wave matches Brillouin frequency of that section fiber, differential Brillouin loss spectrum can be measured, and the improved spatial resolution can be realized at high SNR. By mapping the entire Brillouin frequency ranges of all fiber sections for their BOTDA, so called FDM, we can get the distributed sensing of strain or temperature. The original idea is based on variable Brillouin frequency in fiber to cover a pulse spectrum [44] and Brillouin gain is formed along entire fiber across entire pulse spectrum [45] as shown in Fig. 11. The difference in FDM-BOTDA is that Brillouin gain is only formed at a specific length for a specific Brillouin frequency as shown in Fig. 12.

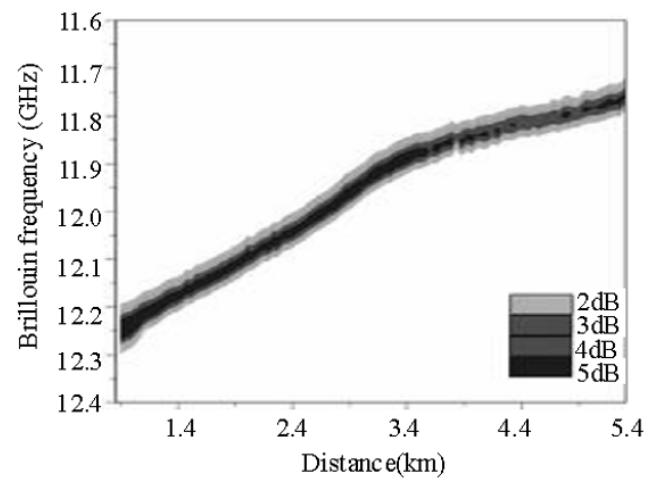

Fig. 11 Brillouin gain spectrum distribution over a fiber [45].

In the experiment, non-uniform fiber with different Brillouin frequency shifts reduces the effective Brillouin amplification length to one resonant Brillouin frequency segment rather than entire sensing fiber, and thus a moderate pump wave 
can be used to enhance Brillouin interaction in an individual segment without pump pulse depletion or excess amplification of probe pulse due to the short Brillouin interaction length for a specific segment. Because of relative short fiber length for the effective sensing fiber length, true Brillouin gain in the section is in fact larger than that over entire sensing length. By using this concept we demonstrated a 75-km BOTDA with three types of $25-\mathrm{km}$ fiber for a spatial resolution of $1.1 \mathrm{~m}$ and an accuracy of $1{ }^{\circ} \mathrm{C} / 20 \mu \varepsilon$ at the end of $75 \mathrm{~km}$, and a spatial resolution of $0.5 \mathrm{~m}$ and an accuracy of $0.7^{\circ} \mathrm{C}$ $/ 14 \mu \varepsilon$ at the end of $50 \mathrm{~km}$. In addition, another $75-\mathrm{km}$ leading fiber also composed of three types of 25-km fiber (listed in Table 2) are used to deliver CW pump to the far end of the sensing fiber, which makes the realization of actual $75-\mathrm{km}$ sensing range, which, to the best of our knowledge, provides the best performance reported so far [46].

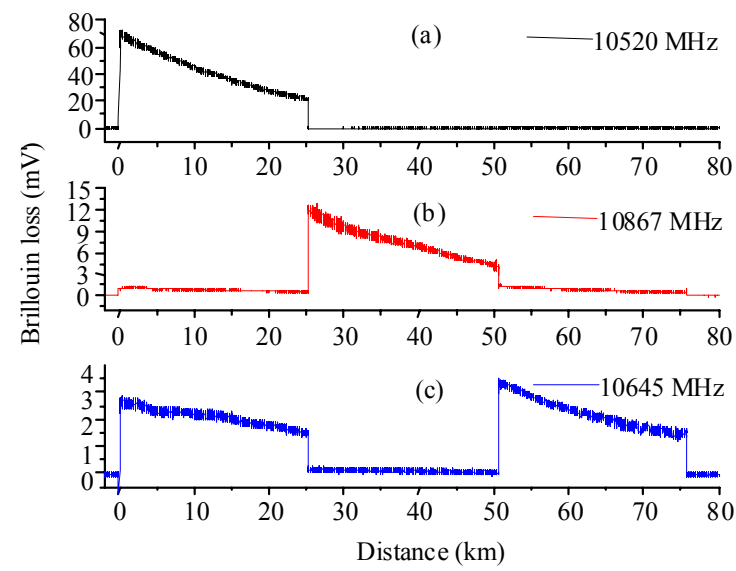

Fig. 12 Illustration time traces of section 1 (a), 2 (b), and 3(c) by FDM [45].

Table $275-\mathrm{km}$ sensing fibers composed of 3 sections.

\begin{tabular}{cccc}
\hline $\begin{array}{c}\text { Fiber } \\
\text { type }\end{array}$ & $\begin{array}{c}g_{B} / A_{\text {eff }} \\
\left(\mathrm{W}^{-1} \cdot \mathrm{m}^{-1}\right)\end{array}$ & $\begin{array}{c}\mathrm{BFS} \\
(\mathrm{MHz})\end{array}$ & $\begin{array}{c}\text { Dispersion } \\
(\mathrm{ps} /(\mathrm{nm} \cdot \mathrm{km}))^{\mathrm{b}}\end{array}$ \\
\hline MetroCor & 0.1669 & 10518 & $-10 \sim-1$ \\
LEAF & 0.0892 & 10645 & $2 \sim 6$ \\
SMF-28 & 0.1281 & 10867 & $16 \sim 19$ \\
\hline
\end{tabular}

a. This is average gain with scrambling the polarization state of probe wave.

b. The values of the dispersion are from datasheet of fibers.

\subsection{TDM based BOTDA}

Here we propose a novel high-performance long-range BOTDA based on TDM, where the sensing range can be extended by multiple measurements of different sections through delay-controlled pump pulse. Furthermore, the system still keeps simple structure without using in-line optical amplifier.

In the proposed scheme two pulses, i.e. a probe pulse and a pump pulse are used to perform the measurement. The spatial resolution is still defined by probe pulse width, while the sensing length is determined by pump pulse width. The delay between the probe pulse and the pump pulse can be changed to select sensing section where the probe pulse interacts with the pump pulse. The measurement of entire sensing fiber is realized by implementing measurement for each sensing section through changing the delay between two pulses, which is named as time-division multiplexing of different fiber sections. Because the interaction length is only determined by the pump pulse width instead of entire fiber, the pump power can be increased to enhance Brillouin interaction in individual section to improve SNR without excess amplification on the probe pulse.

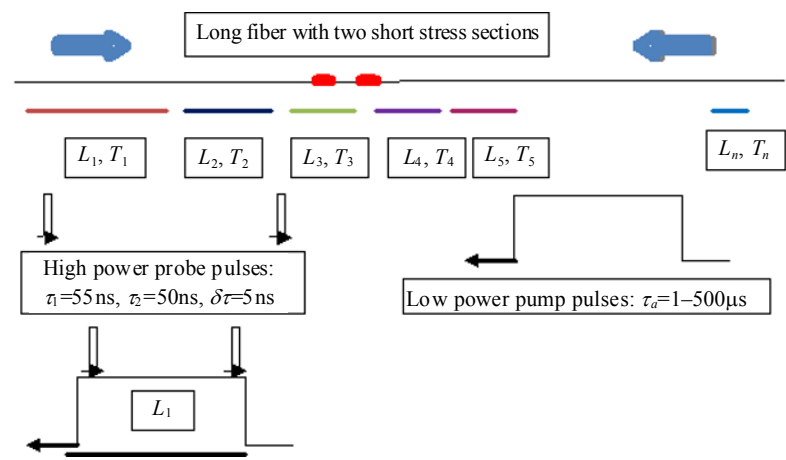

Fig. 13 Time-division-multiplexing for $100-\mathrm{km}$ sensing length.

TDM-BOTDA is most effective on the same fiber type, for instance, SMF28. The effective sensing length is selected by a large pump pulse width as explained in Fig. 13, and differential Brillouin gain spectrum is measured with two smaller pulse widths of probe waves, so that high spatial and temperature resolution can be obtained over entire sensing length. Based on this new 
technique, 0.6-m spatial resolution is achieved at $75-\mathrm{km}$ location and 2-m spatial resolution at $100 \mathrm{~km}$ for Brillion frequency shift measurement accuracy of $1.5 \mathrm{MHz}$. The key to this technique is to maintain equal gain along entire fiber length [47]. Note no inline optical amplifier is used within $100-\mathrm{km}$ sensing length.

\section{Brillouin grating as a point sensor}

Brillouin scattering based fiber sensors can also work as a point sensor. To form a point sensor, one can write a transient Brillouin grating (TBG) [48-51] via two identical optical pulses of frequency difference matching the Brillouin resonance counter-propagating in one of the axes of a PMF. Their overlapping region forms a grating, which can be set at any position in the fiber determined by relative time delay of two pulses, and the size of grating is determined by pulse length. The lifetime of this Brillouin grating is limited by phonon lifetime (10 ns). The grating spectrum is a convolution of pulse spectrum and Brillouin gain spectrum. Such a Brillouin grating can be probed with a 3rd pulse launched on the orthogonal axis; the optimum frequency of the 3rd pulse is related with local birefringence between two axes of PMF. This means the grating feature is affected by temperature and strain reflected in Brillouin frequency and local birefringence from two axes of PMF, and this local TBG can be used for the purpose of sensing. When we change relative delay between two pump pulses a distributed sensing can be realized for entire fiber. Because large frequency shift associated with birefringence change is of the order of $40-50 \mathrm{GHz}$, the measurement accuracy of temperature and strain can be much higher than that in direct Brillouin gain spectrum measurement. It has been reported that $0.08{ }^{\circ} \mathrm{C}$ and $3 \mu \varepsilon$ can be achieved with continuous wave (without location information) [51]. In terms of temperature and strain measurement as a point sensor, TBG is similar to fiber Bragg grating (FBG) sensor, except FBG sensor is a permanent grating, while TBG has a lifetime of $10 \mathrm{~ns}$ with variable length and position determined by writing process and it is a moving grating.

The perturbation of refractive index associated with an acoustic wave can be expressed by

$$
\Delta n=<\delta n>\left[1+\kappa \cos \left(\Omega_{B} t \pm \frac{2 \pi}{\Lambda} z\right)\right]
$$

where $\langle\delta n\rangle$ is average index, and $\kappa$ is fringe visibility of index change, $\Omega_{B}$ is Brillouin frequency, $\Lambda=\lambda / 2 n$ is grating period, and $\lambda$ is vacuum wavelength of pump wave, $n$ is fiber refractive index. This equation is similar to that of fiber Bragg grating except the moving grating term $\left(\cos \left(\Omega_{B} t \pm \frac{2 \pi}{\Lambda} z\right)\right)$ [49]. For the case of Brillouin grating [53], we have the following equation:

$$
\kappa<\delta n>=\frac{\varepsilon_{0} C_{T} \gamma_{e}^{2} E_{P} E_{S}}{2 n}
$$

where $E_{P}$ and $E_{S}$ are electric fields of pump and Stokes waves respectively, $\gamma_{e}$ is electrostriction constant, and $C_{T}$ is compressibility. For a weak Brillouin grating $\left(\kappa<\Delta n><<\frac{\lambda}{L}\right)$, the bandwidth of Brillouin grating is independent of Brillouin scattering linewidth, $\Delta v=\frac{c}{2 n L}$, which has the same relation as that of the Bragg grating. Figure 14 shows the Brillouin grating and its formation by two pump waves with the frequency difference of Brillouin frequency shift. The spectral width of Brillouin grating is illustrated in Fig. 15. In the $x$-axis, two pump pulses of creating gratings are $t_{p 1}$ and $t_{p 2}$.

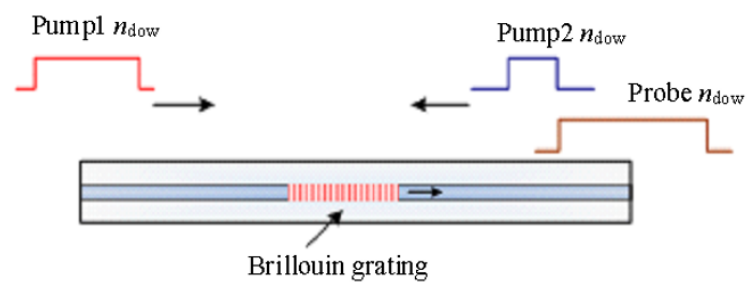

Fig. 14 Brillouin grating generation and reading process.

The moving Brillouin grating and the static fiber Bragg grating follow almost the same theory. The Brillouin grating is created through electrostriction effect and can only be sustained by keeping two 
pump waves. After removing the pump waves, the Brillouin grating will exponentially decay, which characterizes an intrinsic Lorentzian Brillouin gain spectrum. However if the pulse width of pump wave is smaller than the phonon lifetime, the grating spectrum follows Gaussian shape, which reflects the apodization of Brillouin grating feature.

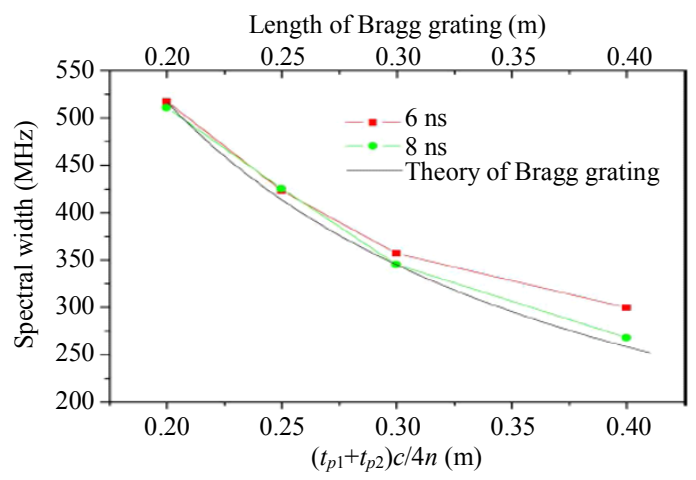

Fig. 15 The intrinsic Brillouin grating spectral width as a function of length [53].

When the grating is formed in one axis of PMF, the probe wave in the other axis can detect Brillouin grating response to temperature and strain via birefringence induced frequency shift between fast and slow axes, so that simultaneous temperature and strain can be realized by Brillouin frequency shift in one axis and birefringence change in the other axis. We realized simultaneous strain and temperature measurement with a high spatial resolution in time domain [50], and a high spatial resolution of $20 \mathrm{~cm}$ is achieved with temperature and strain accuracy of $0.4{ }^{\circ} \mathrm{C}$ and $9 \mu \varepsilon$ and their range up to $700{ }^{\circ} \mathrm{C}$ and $14 \mathrm{me}$.

\section{Dynamic measurement capability with Brillouin scattering}

So far distributed Brillouin sensor has been limited to static strain measurement. In fact one can use polarization dependence of Brillouin gain to measure vibration [54, 55] or dynamic heating process [56] without the need of sweeping Brillouin frequency of the spectrum. Laboratory test has demonstrated distributed measurement of a $13-\mathrm{Hz}$ vibration frequency with 2-m spatial resolution over a 100-m sensing fiber, while for point sensor detection, the maximum vibration frequency of $10 \mathrm{kHz}$ is possible. Such a vibration sensor has been used in the field [54]. Long measurement time of traditional distributed Brillouin sensors is avoided by eliminating frequency sweep of the pump and probe lasers and replaced by locking them at a single beat frequency corresponding to static strain of the structure in which the fiber is embedded. This unique sensor allows distributed measurement of vibration frequencies along a sensing fiber of $300 \mathrm{~m}$ and spatial resolution of $2 \mathrm{~m}$.

\section{Structural health monitoring with distributed sensor based on Brillouin scattering}

Distributed Brillouin sensor is one of the most promising diagnostic tools that could help improve the structural health monitoring process and the first structural strain monitoring using distributed Brillouin sensor was reported in 1999 [57]. Structural health monitoring (SHM) has been used to identify early signs of potential problems of civil structures to prevent disasters, and conduct needed repairs at appropriate time to avoid unnecessary costs and reduce economic burden. Thus it is important to have accurate and real time monitoring on safety assessment of civil structures, such as bridges, dams, and pipelines. The key is to prevent potential disasters. Currently such evaluations are carried out by engineers trained in visual inspection at the risk of sometimes inaccuracy due to personal experience differences on the safety condition assessment generated by this practice. To increase the inspection efficiency and accuracy, fiber optic sensor is one of the most promising candidates due to its features of durability, stability, small sizes, and insensitivity to external perturbations, which makes it ideal for long-term health assessment of civil structures with distributed fiber sensors. Optical fibers can cover large areas of civil structures and 
access the safety and status of the structures. This kind of sensor has the advantages of a long sensing range and the capability of providing strain or temperature at every spatial resolution over entire sensing fiber imbedded in or attached to the structures by using fiber itself as sensing medium $[58,59]$.

Recent studies have been conducted to monitor structural strain of composite and concrete beams under limited load, i.e. the structure responds to the load linearly with average strain over the spatial resolution being monitored [59]. The extraction of average strain is carried with single peak fitting or centroid method (integrated spectrum area method) [58-61]. Those methods provide fast signal processing to identify large strain points, while multiple peak detection [62] provides better strain accuracy with a compromise of long processing time which makes it difficult for dynamic detection and disaster prevention. If the dynamic strain or temperature monitoring is required, intensity detection at fixed Brillouin scattering could be used without scanning Brillouin spectrum [53], which reduces the measurement time significantly. The first few civil structural applications were under elastic working region with the purpose of verifying distributed Brillouin sensor for strain monitoring, in which no deformation happened during loading process. Most of recent research on distributed sensor has been focused on using distributed Brillouin sensor as a tool to correlate the strains to civil structural condition, such as cracks and deformation [57-62], as well as prediction of cracking of concretes and buckling in pipelines using special signal processing schemes via detailed studies of Brillouin spectrum shape change, such as asymmetry broadening in addition to peak change $[57,63]$, as well as cure monitoring of composite materials [64].

Structural health monitoring requires engineers and scientists from different fields to work together to ensure that our civil structures are safe, comfortable and convenient. It is just one of many applications that will benefit the society in terms of the safety of our structures and quality of our lives.

\section{Conclusions}

Fiber sensor based on Brillouin scattering has proven to be a powerful tool for distributed measurements of strain and temperature, and it has been found a number of applications in practice as outlined in previous section. $100-\mathrm{km}$ sensing range is made possible without in-line amplifier inside the sensing length, and for longer distance $(>100 \mathrm{~km})$, the inline amplifiers, such as Raman or EDFA must be considered to compensate fiber loss $[65,66]$. The best spatial resolution is only limited by the resolution of digitizer, for the state of art technology; the time resolution is $50 \mathrm{ps}$, which is equivalent to $20 \mathrm{GHz}$ and $1-\mathrm{cm}$ spatial resolution. The trade-off is low strain or temperature resolution and sensing length, unless current proposed TDM and FDM based BOTDA is employed.

\section{Acknowledgement}

The authors acknowledge the contributions of many of their graduate students, postdoctoral fellows and research associates whose names are listed in reference lists of our paper cited and the supports of the National Science and Engineering Research Council of Canada via Discovery Grants, Equipment Grants and Strategic Grants, and ISIS (Intelligent Sensing for Innovative Structures) Canada, as well as Canada Research Chair Program. Special thank to Shangran Xie for his help in formatting and proof reading.

Open Access This article is distributed under the terms of the Creative Commons Attribution License which permits any use, distribution, and reproduction in any medium, provided the original author(s) and source are credited. 


\section{References}

[1] D. Culverhouse, F. Frahi, C. N. Pannell, and D. A. Jackson, "Potential of stimulated Brillouin scattering as sensing mechanism for distributed temperature sensor," Elect. Lett., vol. 25, no. 14, pp. 913-915, 1989.

[2] T. Horiguchi, T. Kurashima, and M. Tateda, "Tensile strain of Brillouin frequency shift in silica optical fibers," Photon. Tech. Lett., vol. 1, no. 5, pp. 107-108, 1989.

[3] X. Bao, D. J. Webb, and D. A. Jackson, "22 km distributed temperature sensor using Brillouin gain in an optical fiber," Opt. Lett., vo. 18, no. 7, pp. 552-554, 1993.

[4] T. Horiguchi and T. Tateda, "BOTDA-nondestructive measurement of single-mode optical fibers attenuation characteristics using Brillouin interaction: theory," IEEE J. Lightwave Technol, vol. 7, no. 8, pp. 1170-1176, 1989.

[5] M. Nikles, L. Thevenaz, and P. A. Robert, "Simple distributed fiber sensor based on Brillouin gain spectrum analysis," Opt. Lett., vol. 21, no. 10, pp. 758-760, 1996.

[6] X. Bao, D. J. Webb, and D. A. Jackson, "32 km distributed temperature sensor based on Brillouin loss in an optical fiber," Opt. Lett., vol. 18, no. 18, pp. 1561-1563, 1993.

[7] X. Bao, D. J. Webb, and D. A. Jackson, "Combined distributed temperature and strain sensor based on Brillouin loss in an optical fiber," Opt. Lett., vol. 19, no. 2, pp. 141-143, 1994.

[8] X. Bao, J. Dhliwayo, N. Heron, D. J. Webb, and D. A. Jackson, "Experimental and theoretical studies on a distributed temperature sensor based on Brillouin scattering," IEEE J. Lightwave Technol., vol. 13, no. 7, pp. 1340-1348, 1995.

[9] K. Shimizu, T. Horiguchi, Y. Koyamada, and T. Kurashima, "Coherent self-heterodyne detection of spontaneously Brillouin-scattered light waves in a single-mode fiber," Opt. Lett., vol. 18, no. 3, pp. 185-187, 1993.

[10] T. R. Parker, M. Farhadiroushan, V. A. Handerek, and A. J. Rogers, "Temperature and strain dependence of the power level and frequency of spontaneous Brillouin scattering in optical fibers," Opt. Lett., vol. 22, no. 11, pp. 787-789, 1997.

[11] S. M. Maughan, H. H. Kee, and T. P. Newson, "57 km single ended spontaneous Brillouin-based distributed fiber temperature sensor using microwave coherent detection," Opt. Lett., vol. 26, no. 6, pp. 331-333, 2001.

[12] X. Bao, A. Brown, M. DeMerchant, and J. Smith, "Characterization of the Brillouin-loss spectrum of single-mode fibers by use of very short $(<10 \mathrm{~ns})$ pulses," Opt. Lett., vol. 24, no. 8, pp. 510-512, 1999.

[13] V. Lecoeuche, D. J. Webb, C. N. Pannell, and D. A.
Jackson, "Transient response in high-resolution Brillouin-based distributed sensing using probe pulses shorter than the acoustic relaxation time," Opt. Lett., vol. 25, no. 3, pp. 156-158, 2000.

[14] S. Afshar, G. Ferrier, X. Bao, and L. Chen, "The impact of finite extinction ratio of EOM on the performance of the pump-probe Brillouin sensor system," Opt. Lett., vol. 28, no. 16, pp. 1418-1420, 2003.

[15] L. Zou, X. Bao, Y. Wan, and L. Chen, "Coherent pump-probe based Brillouin sensor for $1 \mathrm{~cm}$ crack detection," Opt. Lett., vol. 30, no. 4, pp. 370-372, 2005.

[16] V. P. Kalosha, E. Ponomarev, L. Chen, and X. Bao, "How to obtain high spectral resolution of SBS-based distributed sensing by using nanosecond pulses," Opt. Express, vol. 14, no. 6, pp. 2071-2078, 2006.

[17] T. Horiguchi, K. Shimizu, T. Kurashima, M. Tateda, and Y. Koyamada, "Development of a distributed sensing technique using Brillouin scattering," IEEE J. Lightwave Technol., vol. 13, no. 7, pp. 1296-1302, 1995.

[18] K. Hotate and T. Hasegawa, "Measurement of Brillouin gain spectrum distribution along an optical fiber with a high spatial resolution using a novel correlation-based technique - demonstration of $45 \mathrm{~cm}$ spatial resolution," in 12th International conference on OFS'97-Optical Fiber Sensors, Williamsburg, OSA Technical Digest Series, vol. 16, pp. 337-340, 1997.

[19] K. Hotate and T. Hasegawa, "Measurement of Brillouin gain spectrum distribution along an optical fiber with a high spatial resolution using a correlation-based technique - proposal, experiment and simulation," IEICE Trans. Electron., vol. E83-C, no. 3, pp. 405-411, 2000.

[20] W. Li, X. Bao, Y. Li, and L. Chen, "Different pulse-width pair Brillouin optical time domain analysis (DPP-BOTDR) for high spatial resolution sensing," Opt. Express, vol. 16, no. 26, pp. 21616-21625, 2008.

[21] Y. Li, L. Chen, Y. Dong, and X. Bao, "A novel distributed Brillouin sensor based on optical differential parametric amplification," IEEE J. Lightwave Technol., vol. 28, no. 18, pp. 2621-2626, 2010.

[22] R. W. Boyd, Nonlinear Optics, Second Edition. USA: Academic Press, 2007.

[23] V. Chandrasekharan, "The exact equation for Brillouin shifts," J. Physique, vol. 26, no. 11, pp. 655-658, 1965.

[24] H. I. Mandelberg and L. Witten, "Experimental verification of the relativistic Doppler effect," J. Opt. Soc. Am., vol. 52, no. 5, pp. 529-536, 1962.

[25] A. Minardo, R. Bernini, and L. Zeni, "A Simple 
technique for reducing pump depletion in long-range distributed Brillouin fiber sensors," IEEE Sensor Journal, vol. 9, no. 6, pp. 633-634, 2009.

[26] T. R. Parker, M. Farhadiroushan, R. Feced, and V. A. Habderek, "Simultaneous distributed measurement of strain and temperature from noise-initiated Brillouin scattering in optical fibers," IEEE J. Quantum Electronics, vol. 34, no. 4 pp. 645-659, 1998.

[27] H. K. Huai, G. P. Lees, and T. P. Newson, “All-fiber system for simultaneous interrogation of distributed strain and temperature sensing by spontaneous Brillouin scattering," Opt. Lett., vol. 25, no. 10, pp. 695-697, 2000.

[28] J. Smith, M. DeMerchant, A. Brown, and X. Bao, "Simultaneous distributed strain and temperature measurement," Appl. Opt., vol. 38, no. 25, pp. 5372-5377, 1999.

[29] (a) Q. Yu, X. Bao, and L. Chen, "Temperature dependence of Brillouin frequency, power and bandwidth in Panda, Bow tie and Tiger PM fibers," Opt. Lett., vol. 29, no. 1, pp. 17-19, 2004. (b) Q. Yu, $\mathrm{X}$. Bao, and L. Chen, "The strain dependence of the Brillouin spectrum in polarization maintained fibers at different temperatures," Opt. Lett., vol. 29, no. 14, pp. 1605-1607, 2004.

[30] X. Bao, Q. Yu, and L. Chen, "Simultaneous strain and temperature measurements with PM fibers and their error analysis using distributed Brillouin loss system," Opt Lett., vol. 29, no. 12, pp. 1342-1344, 2004.

[31] L. Zou, X. Bao, and L. Chen, "Study of the Brillouin scattering spectrum in photonic crystal fiber with Ge-doped core," Opt. Lett., vol. 28, no. 21, pp. 2022-2024, 2003.

[32] L. Zou, X. Bao, S. Afshar, and L. Chen, "Dependence of the Brillouin frequency shift on strain and temperature in a photonic crystal fiber," Opt. Lett., vol. 29, no. 13, pp. 1485-1487, 2004.

[33] Y. Li, X. Bao, F. Ravet, and E. Ponomarev, "Distributed Brillouin sensor system based on offset locking of two DFB lasers," Appl. Opt., vol. 47, no. 2, pp. 99-102, 2008.

[34] Y. Doi, S. Fukushima, T. Ohno, and K. Yoshino, "Frequency stabilization of millimeter-wave subcarrier using laser heterodyne source and optical delay line," IEEE Photon. Technol. Lett., vol. 13, no. 9, pp. 1002-1004, 2001.

[35] J. Snoddy, Y. Li, F. Ravet, and X. Bao, "Stabilization of EOM bias voltage drift using lock-in amplifier and PID controller in distributed Brillouin sensor system," App. Opt., vol. 46, no. 9, pp. 1482-1485, 2007.

[36] Y. Dong, L. Chen, and X. Bao, “A high-performance long-range Brillouin loss-based distributed fiber sensor," Appl Opt., vol. 49, no. 27, pp. 5020-5025, 2010.
[37] A. H. Hartog and M. O. Gold, "On the theory of backscattering in single-mode optical fibers," IEEE J. Lightwave Technol., vol. 2, no. 2, pp. 76-84, 1984.

[38] M. A. Soto, G. Bolognini, and F. D. Pasquale, "Analysis of optical pulse coding in spontaneous Brillouin-based distributed temperature sensors," Opt. Express, vol. 16, no. 23, pp. 19097-19111, 2008.

[39] M. A. Soto, G. Bolognini, and F. D. Pasquale, "Enhanced simultaneous distributed strain and temperature fiber sensor employing spontaneous Brillouin scattering and optical pulse coding," IEEE Photon. Technol. Lett., vol. 21, no. 7, pp. 450-452, 2009.

[40] M. A. Soto, G. Bolognini, F. Di Pasquale, and L. Thévenaz "Simplex-coded BOTDA fiber sensor with $1 \mathrm{~m}$ spatial resolution over a $50 \mathrm{~km}$ range," Opt. Lett., vol. 35, no. 2, pp. 259-261, 2010.

[41] N. Linze, W. Li, and X. Bao, "Signal-to-noise ratio improvement in Brillouin sensing," OFS20, Proc. SPIE, vol. 7503, pp. 75036F-1-75036F-4, 2009.

[42] H. Liang, W. Li, N. Linze, L. Chen, and X. Bao, "High resolution DPP-BOTDA over $50 \mathrm{~km}$ fiber using return to zero coded pulses," Opt Lett., vol. 35, no. 10, pp. 1503-1505, 2010.

[43] X. Bao, H. Liang, Y. Dong, W. Li, Y. Li, and L. Chen, "Pushing the limit of the distributed Brillouin sensors for the sensing length and the spatial resolution(Invited Paper)," Proc. SPIE, vol. 7677, pp. 767702, 2010.

[44] V. P. Kalosha, L. Chen, and X. Bao, "Slow light of sub-nanosecond pulses via stimulated Brillouin scattering in non-uniform fibers," Phys. Rev. A: Rapid Communications, vol. 75, no. 2, pp. 21802-21805, 2007.

[45] W. Li, X. Bao, V. P. Kalosha, L. Chen, and M. J. Li, "Using nonuniform fiber to generate slow light via SBS," Research Letters in Optics, vol. 1, Article ID 253634, 4 pages, 2008.

[46] X. Bao, Y. Dong, and L. Chen, "Development and application of the long-range distributed sensors based on Brillouin scattering" in Smart Sensors and Sensing Technology, Ed. Daniel E. Suarez, Nova Scientific Publishers, 2011.

[47] Y. Dong, L. Chen, and X. Bao, "Time-division multiplexing based BOTDA over $100 \mathrm{~km}$ sensing length," Opt Lett., vol. 36, no. 2, pp. 277-279, 2011.

[48] Y. Dong, L. Chen, and X. Bao, "Truly distributed birefringence measurement of polarizationmaintaining fibers based on transient Brillouin grating," Opt. Lett., vol. 35, no. 2, pp. 193-195, 2010.

[49] Y. Dong, X. Bao, and L. Chen, "Distributed temperature sensing based on birefringence effect on transient Brillouin grating in a polarizationmaintaining photonic crystal fiber," Opt. Lett., vol. 34, no. 17, pp. 2590-2592, 2009. 
[50] Y. Dong, L. Chen, and X. Bao, "High-spatial-resolution simultaneous strain and temperature sensor using Brillouin scattering and birefringence in a polarization-maintaining fiber," IEEE Photon. Technol. Lett., vol. 22, no. 18, pp. $1364-1366,2010$.

[51] W. Zou, Z. He, and K. Hotate, "Complete discrimination of strain and temperature using Brillouin frequency shift and birefringence in a polarization-maintaining fiber," Opt. Express, vol. 17, no. 3, pp. 1248-1255, 2009.

[52] T. Erdogan, "Fiber grating spectra," IEEE J. Lightwave Technol., vol. 15, no. 8, pp. 1277-1294, 1997.

[53] Y. Dong, L. Chen, and X. Bao, "Characterization of Brillouin grating spectra," Opt. Express, vol. 18, no. 18, pp. 18960-18967, 2010.

[54] X. Bao, J. Lesson, J. Snoddy, and L. Chen, "Dynamic monitoring of structures, water waves, traffic control, submarine and optical ground wire fibers and intrusion using fiber sensors", in Optical Fiber, New Developments, Ed. Christophe Lethien, InTech, ISBN 978-953-7619-50-3, 2009, Chapter 4, pp. 45-68.

[55] X. Bao, W. Li, C. Zhang, M. Eisa, S. El-Gamal, and B. Benmokrane, "Monitoring the distributed impact wave on concrete slab due to the traffics based on polarization dependence on the stimulated Brillouin scattering," Smart Mater. Struct., vol. 17, no. 1, pp. 15003-15008, 2009.

[56] X. Bao, D. J. Webb, and D. A. Jackson, "Distributed temperature sensor based on Brillouin loss in an optical fiber for transient threshold monitoring," Can J. Phys., vol. 74, no. 1-2, pp. 1-3, 1996.

[57] M. DeMerchant, A. Brown, X. Bao, and T. W. Bremner, "Structural monitoring by use of a Brillouin distributed sensor," Appl. Opt., vol. 38, no. 13, pp. 2755-2759, 1999.

[58]F. Ravet, X. Bao, T. Ozbakaloglu, and M. Saatcioglu, "Signature of structure failure using asymmetric and broadening factors of Brillouin spectrum," IEEE Photon. Technol. Lett., vol. 18, no. 2, pp. 394-396, 2006.

[59] F. Ravet, L. Zou, X. Bao, T. Ozbakkaloglu, M. Saatcioglu, and J. Zhou, "Distributed Brillouin sensor for structural health monitoring," Can. J.
Civil Eng., vol. 34, no. 3, pp. 291-297, 2007.

[60] X. Zeng, X. Bao, C. Y. Chhoa, T. W. Bremner, A. W. Brown, M. DeMerchant, G. Ferrier, A. L. Kalamkarov, and A. V. Georgiades, "Strain measurement in a concrete beam by use of the Brillouin-scattering-based distributed fiber sensor with single-mode fibers embedded in glass fiber reinforced polymer rods and bonded to steel reinforcing bars," Appl. Opt., vol. 41, no. 24, pp. 5105-5114, 2002.

[61] H. Murayama, K. Kageyama, H. Naruse, A. Shimada, and K. Uzawa, "Application of fiber-optic distributed sensors to health monitoring for full-scale composite structures," J. Intell. Mat. Syst. and Struct., vol. 14, no. 1, pp. 3-13, 2003.

[62] (a) A. Deif, B. Cousin, B. Martín-Pérez, C. Zhang, $\mathrm{X}$. Bao, and W. Li, "Concrete deformation in a reinforced concrete beam using distributed Brillouin fiber sensors," Smart Mater. Struct., vol. 19, no. 5, pp. 55014, 2010. (b) B. Martín-Pérez, A. Deif, B. Cousin, C. Zhang, X. Bao, and W. Li, "Strain monitoring in an RC slab sustaining service loads by distributed Brillouin fiber sensors," Can. J. Civ. Eng., vol. 37, no. 10, pp. 1341-1349, 2010.

[63] C. Zhang, X. Bao, I. F. Ozkan, M. Mohareb, F. Ravet, M. Du, and D. J. DiGiovanni, "Prediction of the pipe buckling by using broadening factor with distributed Brillouin fiber sensor," Opt. Fib. Technol., vol. 14, no. 2, pp. 109-113, 2008.

[64] X. Bao, C. Huang, X. Zeng, A. Arcand, and P. Sullivan, "The strain and temperature monitoring of the composite with a Brillouin scattering based distributed fiber sensor," Opt. Eng., vol. 41, no. 7, pp. 1496-1501, 2002.

[65] F. R. Barrios, S. M. Lopez, A. C. Sanz, P. Corredera, J. D. A. Castanon, L. Thevenaz, and M. G. Herraez, "Distributed Brillouin fiber sensor assisted by first-order Raman amplification," IEEE J. Lightwave Technol., vol. 28, no. 15, pp. 2162-2172, 2010.

[66] X. H. Jia, Y. J. Rao, L. Chen, C. Zhang, and Z. L. Ran, "Enhanced sensing performance in long distance Brillouin optical time-domain analyzer based on Raman amplification: theoretical and experimental investigation," IEEE J. Lightwave Technol., vol. 28, no. 11, pp. 1624-1630, 2010. 